The Astrophysical Journal, Vol. 157, July 1969

(C) 1969. The University of Chicago. All rights reserved. Printed in U.S.A.

\title{
OBSERVATIONS OF LOCALIZED 5-MICRON RADIATION FROM JUPITER
}

\author{
J. A. Westphal
}

Division of Geological Sciences, California Institute of Technology, and Mount Wilson and Palomar Observatories, Carnegie Institution of Washington, California Institute of Technology Received June 12, 1969

\section{ABSTRACT}

Localized thermal emission from Jupiter has been observed at $5 \mu$. During April and May 1969 peak brightness temperatures of at least $310^{\circ} \mathrm{K}$ were associated with narrow elongate regions between approximately $5^{\circ}$ and $20^{\circ} \mathrm{N}$. latitude.

\section{INTRODUCTION}

Measurements of 5- $\mu$ radiation from Jupiter by Gillett, Low, and Stein (1969) during 1967 indicate a brightness temperature of approximately $230^{\circ} \mathrm{K}$ from the central region of the planet. These observations represent the first indications of enhanced 5- $\mu$ radiation from Jupiter and stimulated the present investigation.

During the evening of April 21, 1969 (0300 April 22 U.T.), polar scans of Jupiter made with the 200-inch telescope indicated flux levels at an effective wavelength of $4.8 \mu$ greatly exceeding that which could be due to reflected sunlight. On April 25 and 26 and May 14, 15, and 16, 1969, U.T. more complete scanning with small focal-plane apertures indicated that the enhanced radiation at $5 \mu$ was emitted from a discrete dark band between latitudes $5^{\circ}$ and $20^{\circ} \mathrm{N}$. Under conditions of excellent seeing, the detailed $5-\mu$ structure could be correlated with the dark-band structure seen visually.

\section{OBSERVATIONS}

Figure 1, $a$ (Plate L6), illustrates a typical polar scan across Jupiter on April 26, 1969 , with a $3^{\prime \prime}$-diameter round focal-plane diaphragm. An albedo scale constructed by assuming a Lambert flat disk, calibrated from observations of a Leo, is shown along with a monochromatic brightness-temperature scale based on an assumed emissivity of unity. The photograph of Jupiter was taken immediately after the scan, with a camera attached to the photometer. The correlation of the enhanced $5-\mu$ radiation with a prominent dark band is quite striking. The enhanced radiation can be seen to be emitted from the North Equatorial Belt (NEB) (the nomenclature of Peek 1958 is used for the bands and zones of Jupiter).

Scans on May 16, 1969, when the seeing resolution was somewhat better than $1^{\prime \prime}$, show a wealth of detail which correlates directly with the dark structure in the NEB. Figure $1, b$ (Plate L6), is a photograph taken during these scans. A scan with a 1".25 diaphragm along line $A-A^{\prime}$ shows flux emitted over the whole width of the NEB. A similar scan along the line $B-B^{\prime}$ shows two peaks in the $5-\mu$ flux with a value corresponding to an albedo less than unity over the narrow white streamer dividing the band. Scan $B-B^{\prime}$ also detected the only enhanced emission from a region other than the NEB. The intense dark spot just north of the white ovoid area in the South Tropical Zone (STZ) was emitting radiation over a region smaller than $1^{\prime \prime}$ in both dimensions with a flux level about one-half that in the NEB.

On May 14, 1969, observations with a 1".25 diaphragm of the flux from an intensely dark, wedge-shaped area following a white ovoid feature in the border between the $\mathrm{NEB}$ and the Equatorial Zone (EZ) produced the highest flux recorded during these 

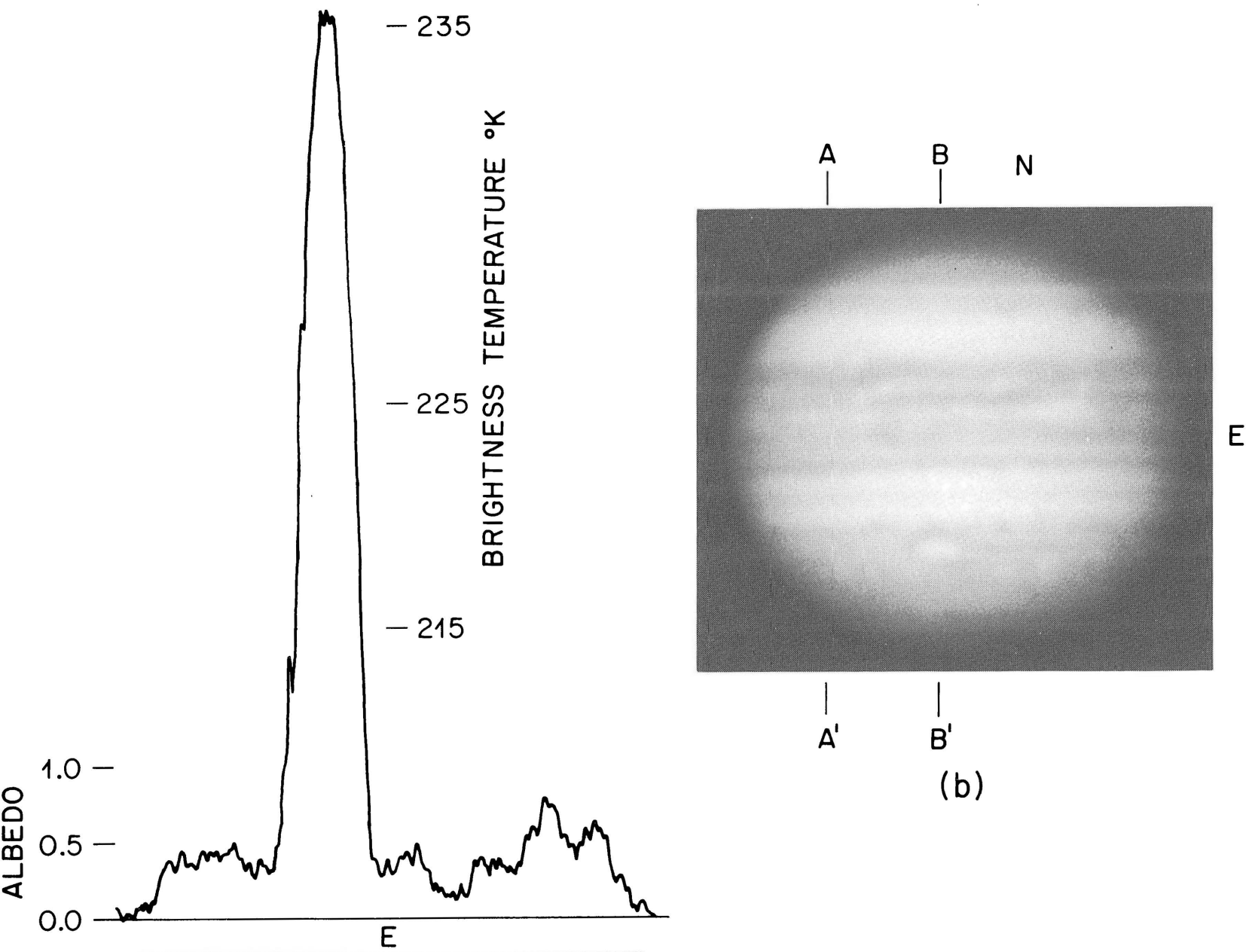

(b)

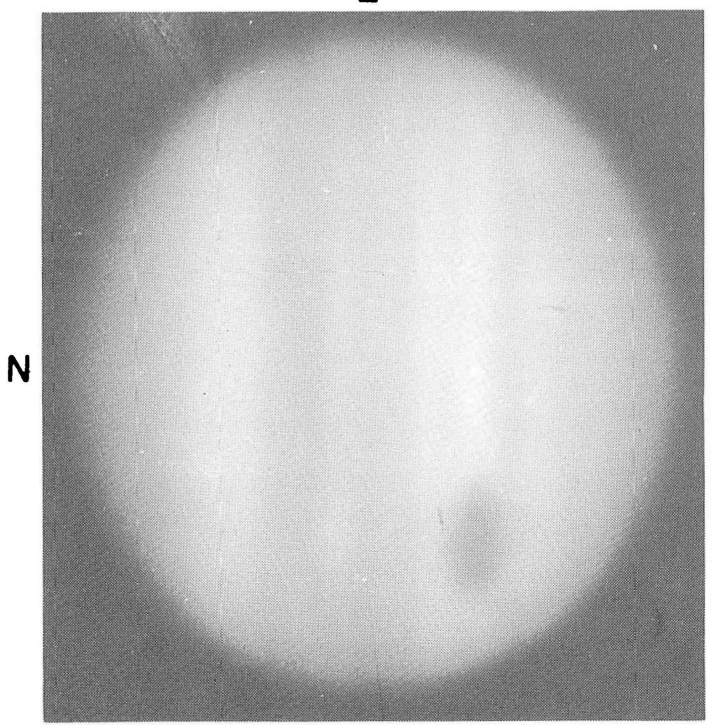

(a)

FIG. 1.-(a) 5- $\mu$ scan along the central meridian of Jupiter on April 22, 1969, U.T. An albedo scale constructed by assuming a Lambert flat disk is shown on the left, and a brightness-temperature scale based on an assumed emissivity of unity is shown on the right. (b) Scan lines $A-A^{\prime}$ and $B-B^{\prime}$ across Jupiter on May 16, 1969, U.T. when the seeing resolution was less than $1^{\prime \prime}$. The polar diameter of Jupiter was $399^{\prime \prime} 3$.

WestPhal (see page L63) 
observations. A brightness temperature of $310^{\circ} \mathrm{K}$ was measured over an extended area of about $2^{\prime \prime} \times 4^{\prime \prime}$.

An examination of all observations during April and May led to the following conclusions:

1. Enhanced radiation at $5 \mu$ was seen on Jupiter in the dark areas of the NEB on all nights.

2. This radiation was present only in the dark areas and not in lighter areas surrounded by these dark areas.

3. Enhanced radiation was seen in only one area, also very dark, outside the NEB.

4. The most intense radiation was seen near the following edge of an ovoid light area in the zone between the NEB and the EZ.

5. No enhancement was seen over the Red Spot.

6 . There were wide variations in the 5- $\mu$ flux at different longitudes along the NEB. Brightness temperatures ranged from $310^{\circ} \mathrm{K}$ downward, but many areas were as hot as $300^{\circ} \mathrm{K}$.

\section{DISCUSSION}

The 5- $\mu$ flux observed from the NEB is much too large to be reflected sunlight from any reasonable scattering material. If the flux is due to thermal emission, it must come from a part of the atmosphere where the local temperature is above $300^{\circ} \mathrm{K}$. Trafton (1967) has calculated model atmospheres which predict temperatures at the top of the convective zone of about $160^{\circ} \mathrm{K}$. If it is assumed that the cloud layer is near the top of the convective zone, this would suggest that the radiation is coming from below the clouds.

If the dark regions of the NEB are locations in which the cloud layer is partially transparent at $5 \mu$, then the observed radiation could originate deep in the atmosphere where the local temperatures are much higher than those at cloud height. Trafton's (1967) model atmospheres predict temperatures of $310^{\circ} \mathrm{K}$ at depths of about $50 \mathrm{~km}$ below the top of the convective zone. However, unless the atmosphere has deeper cloud layers or other discrete layered sources of opacity, it is likely that some of the observed flux comes from even deeper. Indeed, under the very best seeing conditions, the NEB is seen to consist of many very small, intensely dark spots with lighter surrounding areas. Thus the measured flux is often an average over several of these small spots, and the calculated brightness temperature may be much too low if all the radiation is coming from the unresolved dark spots. This is illustrated by scans similar to that in Figure $1, a$, where the measured brightness temperatures in a 3 ".5 diaphragm were only around $240^{\circ} \mathrm{K}$, while repeat scans over the same area with a 1 .'25 diaphragm indicated small spots with brightness temperatures of greater than $300^{\circ} \mathrm{K}$.

Since, in the visible, the NEB is darker than any other feature on Jupiter (McCord 1969), the possibility exists that this band is relatively free of clouds at these wavelengths and that the atmosphere of Jupiter in the NEB is transparent at all wavelengths where gaseous absorption is not severe. The clearest regions seem to be adjacent to ovoid features. Perhaps the physical mechanism that forms these features also causes unusual clearing of the thin clouds in the immediate vicinity.

I would like to thank Drs. G. Neugebauer, P. Goldreich, and A. Ingersoll for valuable discussions. This work was supported in part by NASA grant NGL 05-002-003.

\section{REFERENCES}

Gillett, F. C., Low, F. J., and Stein, W. A., 1969 (in press).

McCord, T. B. 1969 (private communication).

Peek, B. M. 1958, The Planet Jupiter (London: Faber \& Faber).

Trafton, L. M. 1967, Ap. J., 147, 765. 